\title{
Pharmacokinetics in Zebrafish Embryos (ZFE) Following Immersion and Intrayolk Administration: A Fluorescence-Based Analysis
}

\author{
Marlly Guarin ${ }^{1}\left(\mathbb{D}\right.$, Annelii Ny ${ }^{1}$ D , Noémie De Croze ${ }^{2}$, Jan Maes ${ }^{1}$, Marc Léonard ${ }^{2}$, Pieter Annaert ${ }^{3, *(D)}$ \\ and Peter A. M. de Witte ${ }^{1, *(1)}$ \\ 1 Laboratory for Molecular Biodiscovery, Department of Pharmaceutical and Pharmacological Sciences, \\ University of Leuven, 3000 Leuven, Belgium; marlly.guarin@kuleuven.be (M.G.); \\ annelii.ny@kuleuven.be (A.N.); jan.maes@kuleuven.be (J.M.) \\ 2 L'Oréal Research \& Innovation, 93600 Aulnay-sous-Bois, France; noemie.decroze@rd.loreal.com (N.D.C.); \\ marc.leonard@rd.loreal.com (M.L.) \\ 3 Drug Delivery and Disposition, Department of Pharmaceutical and Pharmacological Sciences, \\ University of Leuven, 3000 Leuven, Belgium \\ * Correspondence: pieter.annaert@kuleuven.be (P.A.); peter.dewitte@kuleuven.be (P.A.M.d.W.)
}

Citation: Guarin, M.; Ny, A.; De Croze, N.; Maes, J.; Léonard, M.; Annaert, P.; de Witte, P.A.M.

Pharmacokinetics in Zebrafish Embryos (ZFE) Following Immersion and Intrayolk Administration: A Fluorescence-Based Analysis. Pharmaceuticals 2021, 14, 576. https://doi.org/10.3390/ph14060576

Academic Editor: Marc Ekker

Received: 28 May 2021

Accepted: 13 June 2021

Published: 16 June 2021

Publisher's Note: MDPI stays neutral with regard to jurisdictional claims in published maps and institutional affiliations.

Copyright: (c) 2021 by the authors. Licensee MDPI, Basel, Switzerland. This article is an open access article distributed under the terms and conditions of the Creative Commons Attribution (CC BY) license (https:/ / creativecommons.org/licenses/by/ $4.0 /)$.

\begin{abstract}
Zebrafish embryos (ZFE) have increasingly gained in popularity as a model to perform safety screenings of compounds. Although immersion of ZFE is the main route of exposure used, evidence shows that not all small molecules are equally absorbed, possibly resulting in false-negative readouts and incorrect conclusions. In this study, we compared the pharmacokinetics of seven fluorescent compounds with known physicochemical properties that were administered to two-cell stage embryos by immersion or by IY microinjection. Absorption and distribution of the dyes were followed at various timepoints up to $120 \mathrm{hpf}$ by spatiotemporal fluorescence imaging. The concentration $(10 \mu \mathrm{M})$ and dose $(2 \mathrm{mg} / \mathrm{kg})$ used were selected as quantities typically applied in preclinical experiments and zebrafish studies. The data show that in the case of a lipophilic compound $(\log$ D: 1.73) the immersion procedure resulted in an intrabody exposure which is similar or higher than that seen after the IY microinjection. In contrast, zero to low intrabody exposure was reached after immersion of the embryos with less lipophilic compounds. In the latter case IY microinjection, a technical procedure that can be easily automated, is highly recommended.
\end{abstract}

Keywords: zebrafish embryo; fish embryo toxicity test (FET); pharmacokinetics; non-compartmental analysis; fluorescent compounds; exposure routes; false-negatives; absorption; distribution; computational biology

\section{Introduction}

Zebrafish embryos (ZFE) have increasingly gained in popularity as a model to perform safety screenings of compounds. Due to their small size and transparent bodies, which enable testing of compounds in 96-384 microwell plates and assessment of phenotypes using a dissecting microscope, the ZFE model combines the high throughput capability of in vitro systems with the physiological complexity of a whole vertebrate organism $[1,2]$. Moreover, this model adheres to the 3Rs principle of animal experimentation by providing refinement and partial replacement over higher animal models [1].

Efforts have been made to standardize and validate the use of ZFE in toxicity evaluations for regulatory purposes, the most common one being the fish embryo toxicity test (FET) defined in guideline 236 of the OECD (Organization for Economic Cooperation and Development). FET involves the use of zebrafish to determine the acute toxicity of chemicals during their embryonic stages by exposing newly fertilized eggs to test chemicals dissolved in their surrounding medium for $96 \mathrm{hpf}$ [3]. Busquet and collaborators [4] evaluated the reproducibility of this zebrafish-based assay and identified it as being a 
robust screening method, in general providing acceptable repeatability and reproducibility rates with a coefficient of variation $<30 \%$.

However, in the case of very toxic, volatile, or poorly soluble chemicals, the coefficient variation was $>30 \%$. This outcome highlights the importance of compound's physicochemical properties for the uptake and intrabody distribution of substances tested. In fact, although immersion of ZFE is the main route of exposure used, evidence shows that not all small compounds are equally absorbed by ZFE. Furthermore, the bioavailability of a compound may also vary depending on the developmental stage of the ZFE at the initiation of the experiment [5].

Actually, compounds face two barriers before being taken up by the ZFE, i.e., the chorion, a N-linked glycoprotein envelope pierced by circular pores of a diameter of $0.5-1.5 \mu \mathrm{m}$, and the vitelline bilayer membrane that surrounds directly the embryo and yolk [6]. The chorion is known to affect chemical uptake, depending on the molecule size, ionic charge, and the physicochemical properties of the molecule, among other parameters $[7,8]$. Consequently, embryos have been dechorionated before exposure to chemicals to improve the sensitivity towards toxicants of the zebrafish-based testing platforms [9].

Some pharmaceuticals seem however to pass readily the chorion and accumulate in the perivitelline space between the chorion and the vitelline membrane [7]. A study exposing embryos to hydrophilic dyes, showed that $90 \%$ of compound present in the egg was not localized within the embryo or chorion but rather distributed into the perivitelline space [10]. In contrast, lipophilic dyes were taken up readily [10]. These results show that the membrane and not the chorion can form an obstacle for intrabody exposure to some chemicals. A similar explanation has been cited to account for the lack of activity of cryoprotectants for fish eggs and embryos [11]. Whatever the reason, a relative lack of absorption might result in false-negative readouts and lead to incorrect conclusions in safety assessment evaluations. To avoid issues related to immersion, administration of compounds by parenteral exposure, i.e., intrayolk microinjection (IY), has been implemented in young embryos [12]. However, data demonstrating how compounds distribute in ZFE after IY delivery is lacking. Moreover, a detailed comparison with results using the same chemicals obtained by the immersion route, and relating the data with their physicochemical characteristics, is missing.

In this study, we compared the pharmacokinetics of seven fluorescent compounds with known physicochemical properties that were administered to two-cell stage embryos by immersion or by IY microinjection. Absorption and distribution of the dyes were examined at various timepoints after administration by spatiotemporal fluorescence imaging. Relative exposure levels were first calculated using noncompartmental pharmacokinetic analysis and then related to some physicochemical descriptors of the compounds.

\section{Results}

\subsection{Spatiotemporal Imaging Following Immersion and IY Microinjection}

Immersion: zebrafish embryos at the two-cell stage were immersed in a $10 \mu \mathrm{M}$ solution of each of the seven fluorescent dyes for a maximum of $72 \mathrm{~h}$. Afterwards the embryos were kept for another $48 \mathrm{~h}$ in Danieau's medium in the absence of compound. At specific time points, the embryos were immobilized, and fluorescent pictures taken. As seen in Figure 1, embryos were highly fluorescent after $1 \mathrm{~h}$ immersion, but in most cases the fluorescence was lost after removal of the chorion and rinsing of the embryo, except in the case of CY3A. Longer incubations (24-72 h) resulted in a further intrabody accumulation of the compound, as also observed in the case of TAMRA. During the wash-out phase (72-120 h) some redistribution of the fluorescence could be observed.

IY microinjection: each of the compounds $(2 \mathrm{mg} / \mathrm{kg}$ ) was administered in zebrafish at the two-cell stage by microinjection in the yolk sac. In some embryos we could observe a flow of the dye from the injection site towards the proliferating embryonal cells during the first hours post injection (Supplementary Figure S1). However, a significant part of the 
compounds stayed localized to the microinjection site, especially in the case of dyes with a low Log D value, i.e., S-CY3A, S-CY5.5A and S-CY5A. At later time points, the fluorescent molecules distributed in the yolk area and also in the rest of the body, particularly in the 48-72 $\mathrm{h}$ period (Figure 2). Of interest, we also noticed an accumulation of S-CY3A, FAMA, TAMRA, R6GA, and CY3A in the gastrointestinal system after $96 \mathrm{hpf}$, and an excretion of some compounds by means of the cloaca that is functional at this developmental stage [13]. The spatiotemporal distribution of the fluorescent dyes in the embryos shows that the average fluorescence levels reached are substantially lower during immersion exposure than after microinjection, except for compound CY3A (Figure 2).

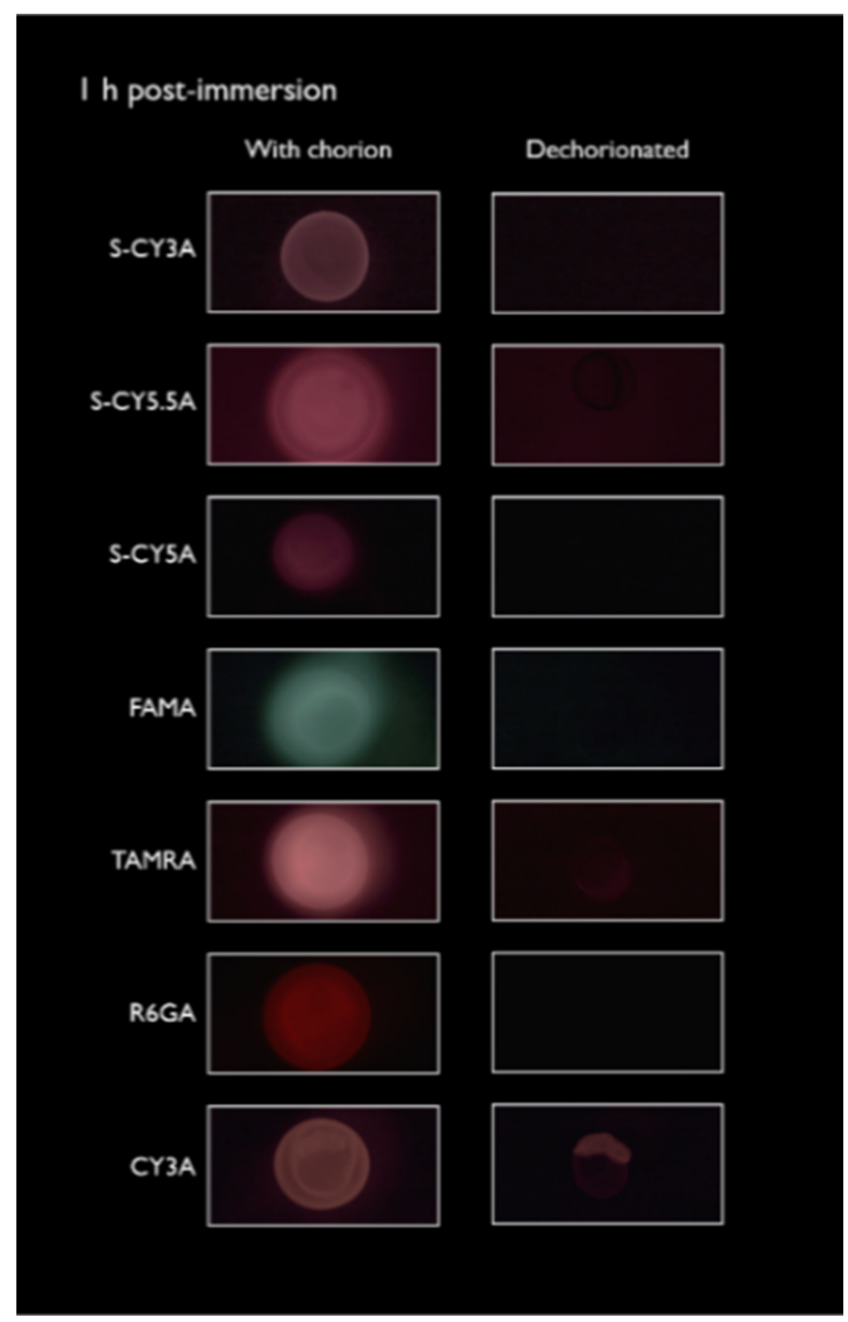

Figure 1. Representative fluorescent images of embryos exposed to the different fluorescent compounds by immersion $(10 \mu \mathrm{M})$ for $1 \mathrm{~h}$, before (left panel) and after dechorionation and rinsing (right panel). 


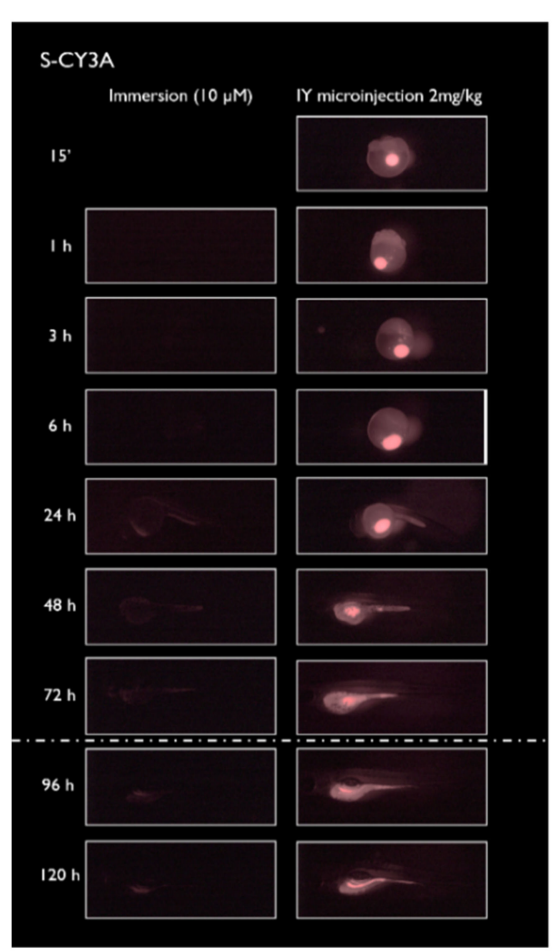

(a)

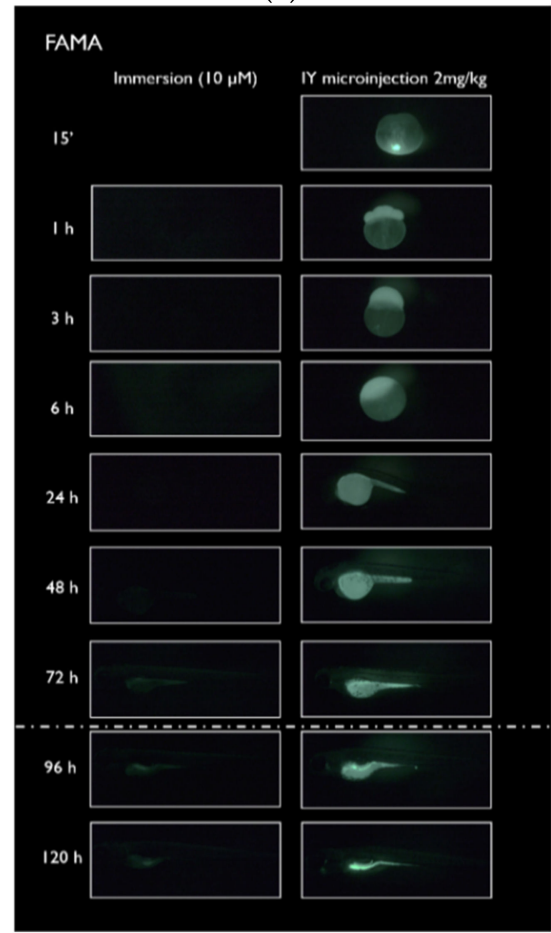

(d)

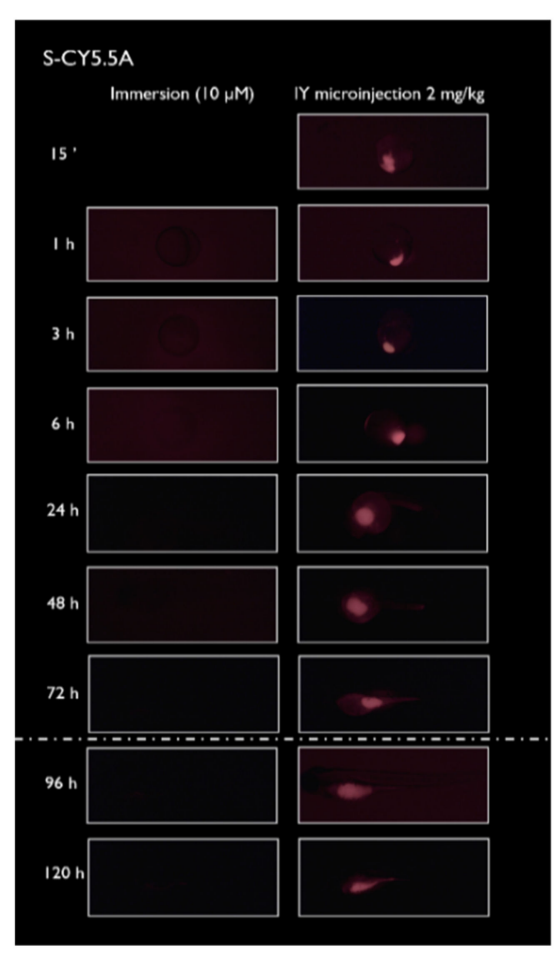

(b)

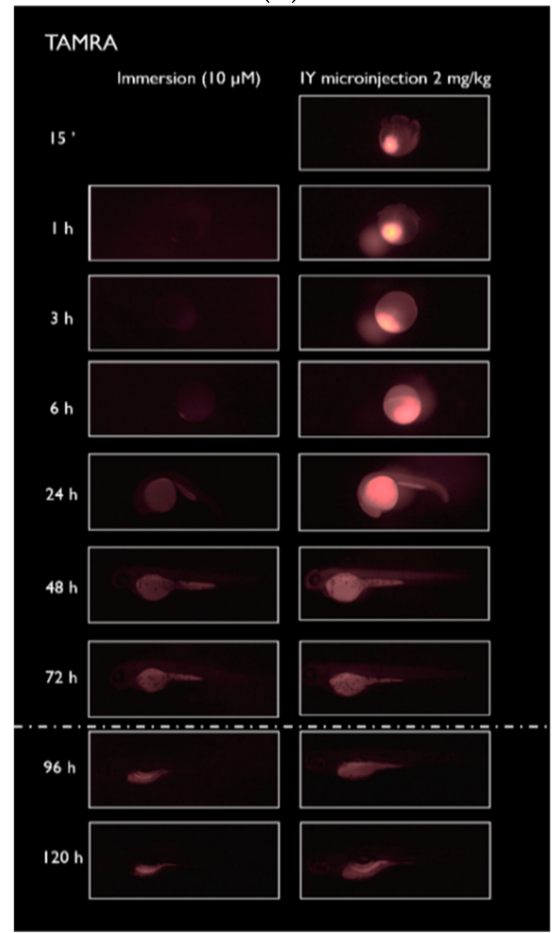

(e)

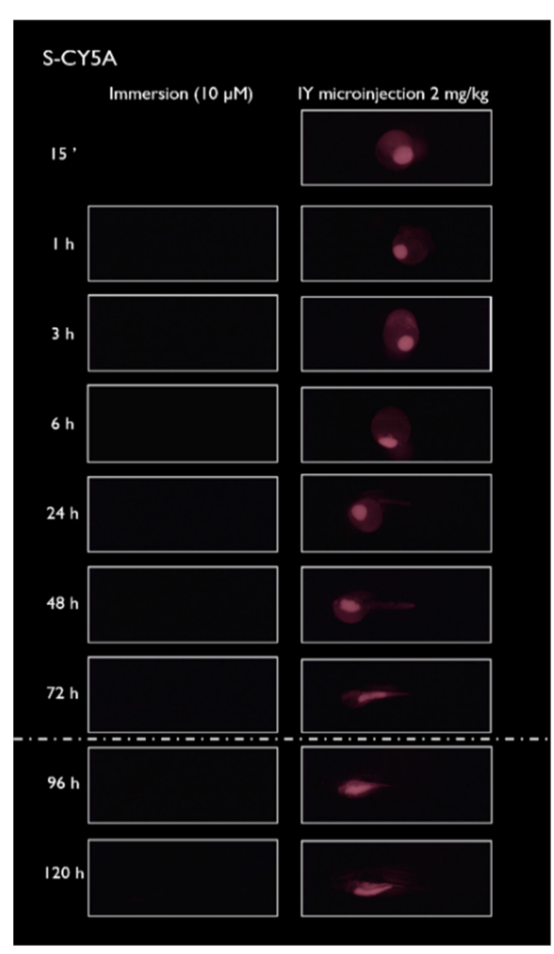

(c)

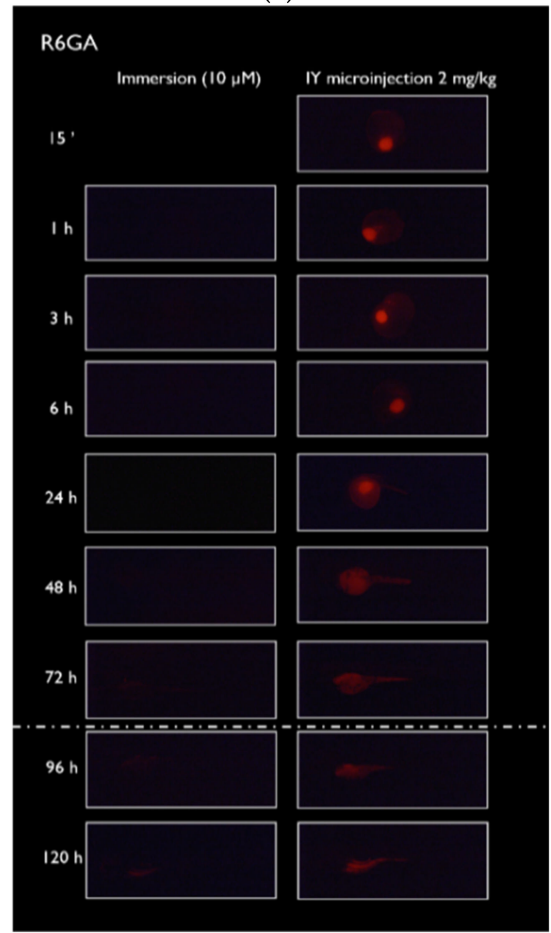

(f)

Figure 2. Cont. 


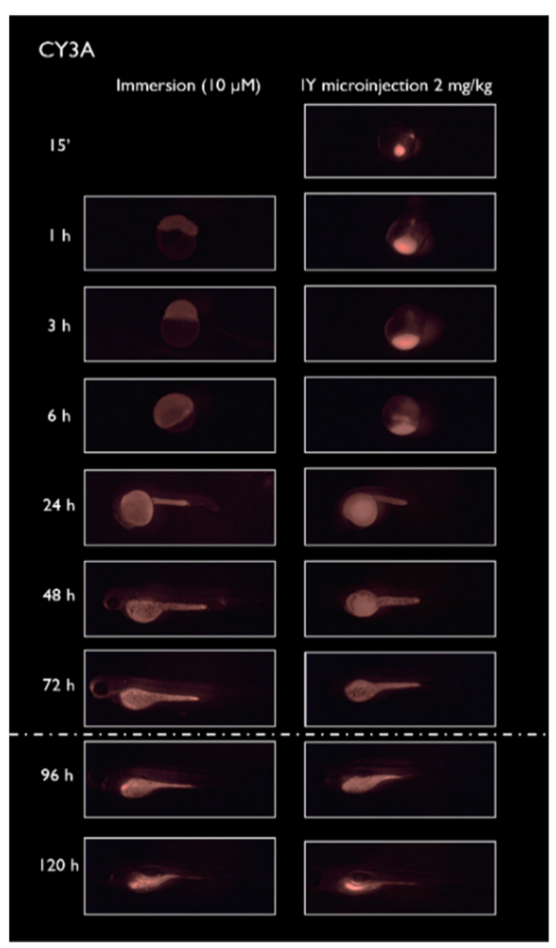

$(\mathrm{g})$

Figure 2. Representative fluorescent images showing the spatiotemporal distribution of the fluorescent dyes in the embryos after immersion $(10 \mu \mathrm{M})$ and microinjection $(2 \mathrm{mg} / \mathrm{kg})$ for several periods of time. (a) animals treated with S-CY3A; (b) S-CY5.5A; (c) S-CY5A; (d) FAMA; (e) TAMRA; (f) R6GA; (g) CY3A. The dash dotted line represents the hatching time and the change of zebrafish medium. $15^{\prime}=0.25 \mathrm{~h}$.

\subsection{Non-Compartmental Pharmacokinetic Analysis}

Next, we calculated the relative amount of fluorescent dye present in the embryos from 0.25 to $72 \mathrm{~h}$ post exposure by quantification of the integrated fluorescent intensity in delineated whole-body contours. Of notice, the maximal fluorescence intensity was not recorded immediately after microinjection, probably by temporal formation of aggregates that show no fluorescence during early time points [14]. These early observations were therefore censored per compound and excluded from the dataset. The semilogarithmic graphs in Figure 3a show that only in the case of CY3A the immersion and intrayolk microinjection procedure resulted in a similar exposure profile.

In addition, as the yolk makes up a significant portion of the zebrafish embryo during the first stages of development and is known to retain small molecules due to its high content of cholesterol and triacylglycerol [15], we also assessed the amount of fluorescent dye present in the yolk and in the rest of the body (RoB, i.e., non-yolk parts). We only measured up to $72 \mathrm{hpf}$ as the yolk part becomes difficult to discern from the rest of the body in later periods. Figure $3 \mathrm{~b}$ shows that after IY microinjections the amount of fluorescent dye present in the yolk was higher than in the RoB parts for all compounds during the whole time. In the case of immersions, the accumulation of the fluorescent dyes in yolk and RoB was less divergent. 


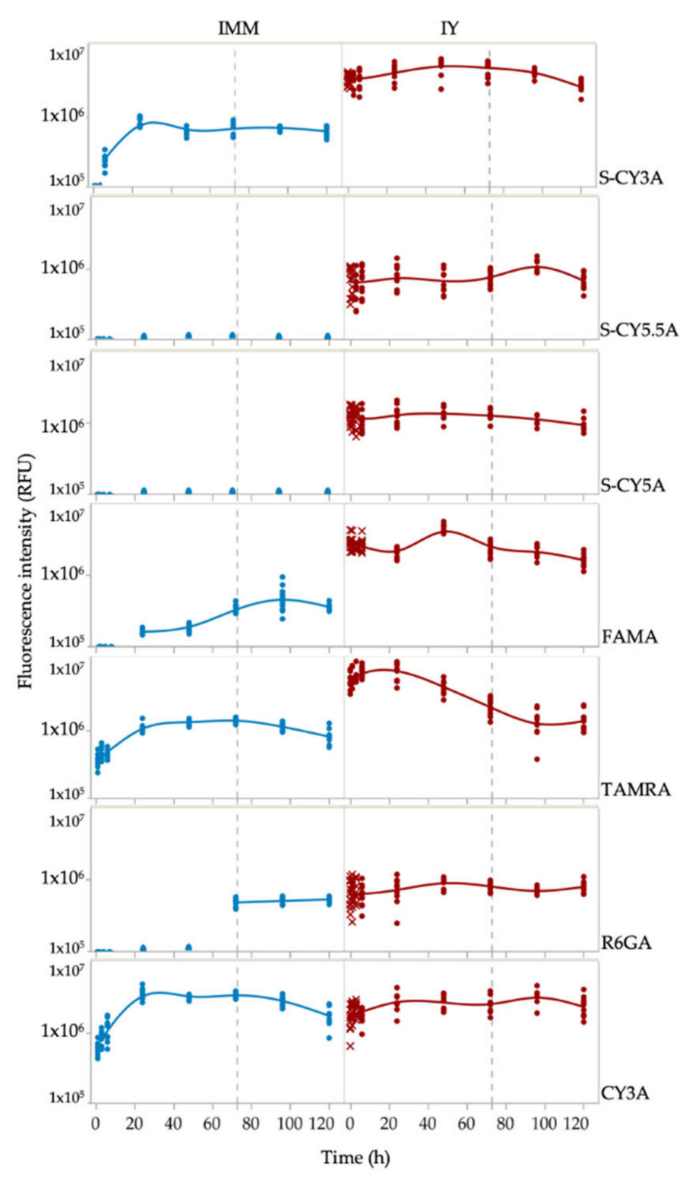

(a)

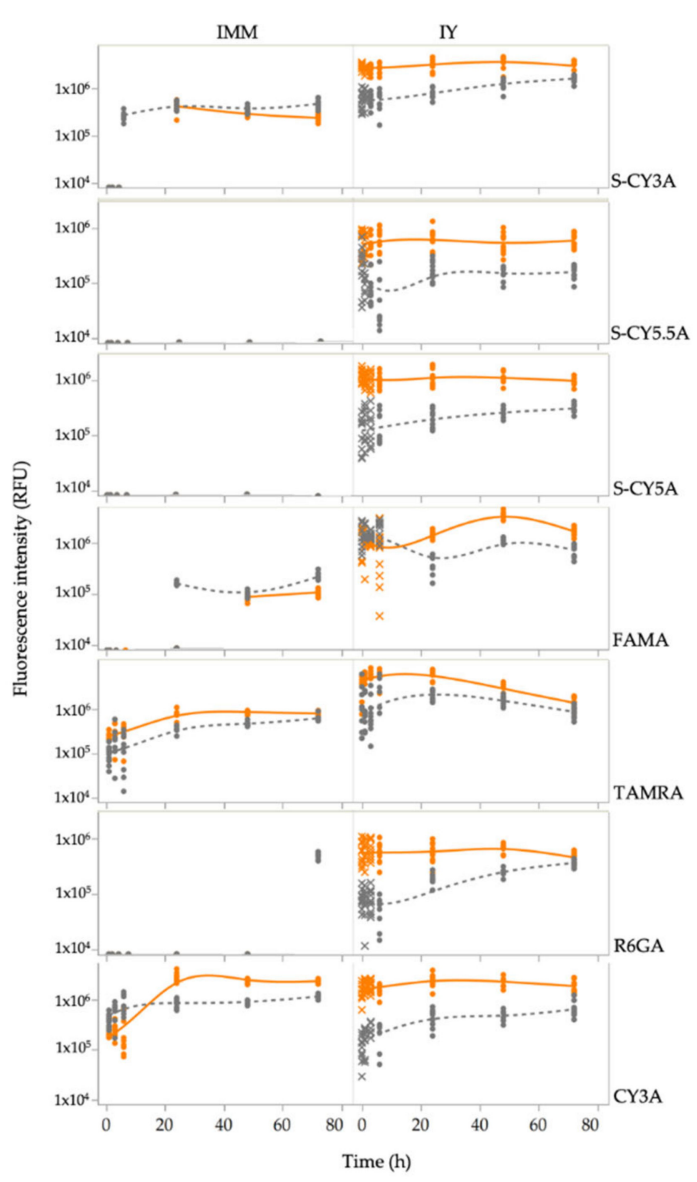

(b)

Figure 3. Semilogarithmic graphs of the fluorescence intensity (RFU) as a function of time, in the case of immersion (IMM) and intrayolk (IY) exposure routes (a) in the whole-body (from 0.25 to $120 \mathrm{~h}$ ). The dashed line at $72 \mathrm{~h}$ indicates the start of the rinsing period. (b) in the yolk (orange line) and non-yolk parts (RoB, Rest of Body) (gray dash line) (from 0.25 to $72 \mathrm{~h}$ exposure). Excluded data points are marked as X symbols.

We then assessed by non-compartmental analysis the area from 0.25 to $72 \mathrm{~h}$ post exposure under the concentration-time curve $\left(\mathrm{AUC}_{0.25-72 \mathrm{~h}}\right)$ (Table 1$)$ that represents well the relative tissue exposure to fluorescent compound across time. These absolute fluorescence intensities however cannot be comscriptpared across compounds as they depend critically on the respective quantum yields of the compounds and the device settings. To be able to compare the PK behavior of the compounds following different administration routes, we calculated the fluorescence-independent pharmacokinetic parameter Relative Exposure $\left(\mathrm{RE}_{10 / 2 / 72 \mathrm{~h}}\right)$, i.e., the ratio of $\mathrm{AUC}_{0.25-72 \mathrm{~h}}$ immersion $(10 \mu \mathrm{M})$ to $\mathrm{AUC}_{0.25-72 \mathrm{~h}} \mathrm{IY}$ microinjection $(2 \mathrm{mg} / \mathrm{kg})$. Separate RE values were calculated based on the whole body (WB), rest-of-body (RoB) and yolk fluorescence measurements (Table 2).

The results show that the RE-WB $\mathrm{W}_{10 / 2 / 72 \mathrm{~h}}$ was low $(0.05-0.19)$ for most compounds, except for CY3A (RE-WB $10 / 2 / 72 \mathrm{~h}$ : 1.11). A similar outcome was obtained for RE-RoB ${ }_{10 / 2 / 72 h}$, although S-CY3A and CY3A displayed values that were two-fold as high as the corresponding RE$\mathrm{WB}_{10 / 2 / 72 \mathrm{~h}}$ results. The data indicate that tissue exposure was 3- to 9-fold higher for most compounds as a function of time when administered by microinjection than after immersion, except in the case of CY3A (i.e., two-fold higher after immersion). The RE-Yolk $10 / 2 / 72 \mathrm{~h}$ values are low to very low for most compounds, except for Cy3A, demonstrating that non-lipophilic compounds are not readily taken up by yolk after immersion. 
Table 1. $\mathrm{AUC}_{0.25-72 \mathrm{~h}}$ values calculated following immersion $(10 \mu \mathrm{M})$ and intrayolk microinjection $\left(2 \mathrm{mg} / \mathrm{kg}\right.$ ) from 0.25 to $72 \mathrm{~h}$ for the whole-body (WB), yolk and RoB parts. $\mathrm{AUC}_{0.25-72 \mathrm{~h} \text { values }}$ expressed as RFU $\times 10^{7}$.

\begin{tabular}{ccccccc}
\hline \multirow{2}{*}{ Compound } & \multicolumn{2}{c}{ AUC-WB } & \multicolumn{2}{c}{ AUC-Yolk } & \multicolumn{2}{c}{ AUC-RoB } \\
\cline { 2 - 7 } & IMM & IY & IMM & IY & IMM & IY \\
\hline S-CY3A & 4.56 & 31.4 & 1.89 & 23.8 & 2.67 & 7.65 \\
S-CY5.5A & 0 & 5.45 & 0 & 4.42 & 0 & 1.02 \\
S-CY5A & 0 & 9.49 & 0 & 7.82 & 0 & 1.67 \\
FAMA & 1.17 & 27.8 & 0.36 & 14.9 & 0.84 & 6.93 \\
TAMRA & 7.76 & 40.4 & 4.98 & 29.1 & 2.78 & 11.2 \\
R6GA & 0.58 & 5.76 & 0 & 4.29 & 0.58 & 1.48 \\
CY3A & 20.9 & 18.9 & 14.6 & 15.7 & 6.41 & 3.21 \\
\hline
\end{tabular}

Based on the $\mathrm{AUC}_{0.25-72 \mathrm{~h}}$ results, we further calculated the Relative Distribution (RD) after microinjection, i.e., the ratio of $A U C_{R o B}$ to $A U C_{W B}$ after microinjection of the compounds into the yolk $(2 \mathrm{mg} / \mathrm{kg})$. The data show that all microinjections resulted in rather low RD values, ranging from 0.17 to 0.32 (Table 2), indicating that only a partial amount of the dose injected was redistributed during the first $72 \mathrm{~h}$ to the non-yolk parts of the ZFE.

Table 2. Relative exposure ( $R E$ ) values (WB: whole body, RoB: rest of body) in the case of immersion and intrayolk microinjection and relative distribution ratio $(R D)$ after microinjection.

\begin{tabular}{ccccc}
\hline Compound & $\boldsymbol{R E}-\boldsymbol{W B}$ & $\boldsymbol{R E - R o B}$ & $\boldsymbol{R E}$-Yolk & $\boldsymbol{R D}(\mathrm{IY})$ \\
\hline S-CY3A & 0.15 & 0.35 & 0.08 & 0.24 \\
S-CY5.5A & 0 & 0 & 0 & 0.19 \\
S-CY5A & 0 & 0 & 0 & 0.18 \\
FAMA & 0.05 & 0.12 & 0.02 & 0.32 \\
TAMRA & 0.19 & 0.25 & 0.17 & 0.28 \\
R6GA & 0.10 & 0.39 & 0 & 0.26 \\
CY3A & 1.11 & 1.99 & 0.93 & 0.17 \\
\hline
\end{tabular}

\subsection{QSPkR Analysis}

Next, we performed a QSPkR (Quantitative Structure-Pharmacokinetic Relationship) analysis to correlate calculated RE values with a set of molecular descriptors of the fluorescent dyes used (Table 3). Through multiple regression modelling (MLR) we identified a parabolic relationship between $\mathrm{RE}_{\mathrm{WB}}$ and $\mathrm{RE}_{\mathrm{RoB}}$, and $\log \mathrm{D}\left(\mathrm{R}^{2} 0.81\right.$, RMSE $0.213, p<0.037 ; \mathrm{R}^{2} 0.82$, RMSE 0.299, P 0.015; respectively) (Figure 4). In the case of $R E_{Y o l k}$, the outcome also included other descriptors, i.e., TPSA, MR and MR ( $\mathrm{R}^{2} 0.99$, RMSE 0.043, $p$ 0.011). The RMSE values of the $R E_{W B}$ and $R E_{R o B}$ models are in the range of observed $R E$ values for compounds with Log $D$ values below 1 . As illustrated in Figure 4, this is consistent with comparable RE values for $6 / 7$ compounds tested in the range $-2<\log \mathrm{D}<1$. Nevertheless, the models clearly predict substantial increase in these RE values for compounds with $\log D$ values above the cut-off of 1.5. In addition, adequate correlations between the RE values and Log D values are also reflected by the corresponding $R^{2}$ and $p$-values (see Table 3 ). 


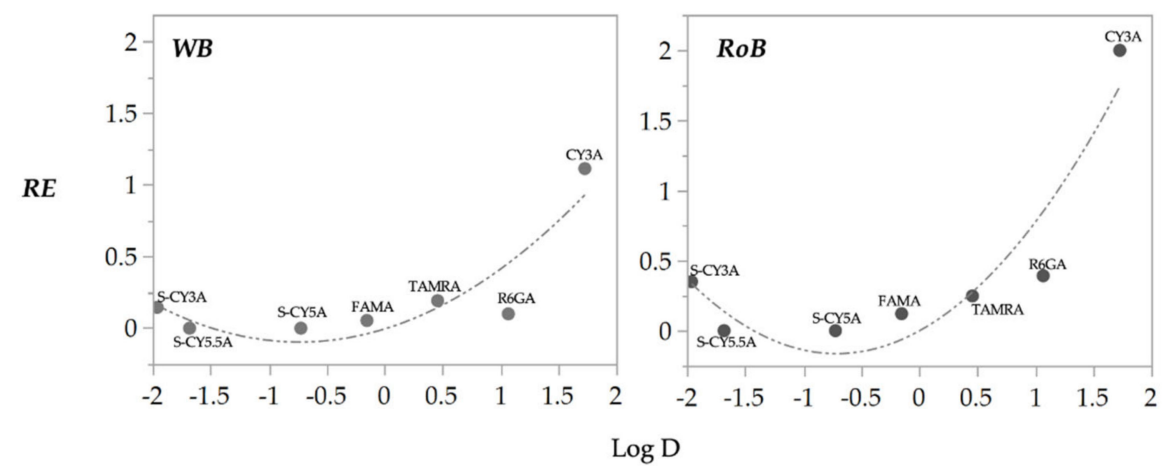

Figure 4. Relationship between observed RE and Log D for the WB (left) and the RoB (right) of the 7 fluorescent compounds. The dashed line is line of fit.

Table 3. QSPkR analysis results. MLR of the $R E$ and molecular descriptors as explanatory variable of the models. Only statistically significant models are shown. adj: $\mathrm{R}^{2}$ adjusted. RMSE: root mean square error. $p$-Value $(<0.05)$.

\begin{tabular}{|c|c|c|c|c|}
\hline PK. Parameter & Model & $R^{2}$ adj & RMSE & $p$-Value \\
\hline $\mathrm{RE}_{W B 10 / 2 / 72 \mathrm{~h}}$ & $=-0.011+0.187(\log \mathrm{D})+(\log \mathrm{D}+0.179)^{2} \times 0.169$ & 0.81 & 0.213 & 0.037 \\
\hline $\mathrm{RE}_{\mathrm{RoB} 10 / 2 / 72 \mathrm{~h}}$ & $=-0.013+0.340(\log \mathrm{D})+(\log \mathrm{D}+0.179)^{2} \times 0.320$ & 0.82 & 0.299 & 0.015 \\
\hline $\mathrm{RE}_{Y o l k 10 / 2 / 72 \mathrm{~h}}$ & $\begin{array}{l}=-1.473+0.313(\log \mathrm{D})+0.006(\mathrm{TPSA})+0.009(\mathrm{MR})+(\log \mathrm{D}+0.179) \\
(\mathrm{MR}-166.591) \times 0.015\end{array}$ & 0.99 & 0.043 & 0.011 \\
\hline
\end{tabular}

\section{Discussion}

In this study we compared the pharmacokinetics of seven photostable fluorescent compounds encompassing Log D values from -1.96 to 1.73 in zebrafish embryos after immersion or microinjection. The concentration $(10 \mu \mathrm{M})$ and dose $(2 \mathrm{mg} / \mathrm{kg})$ used were selected as quantities typically applied in preclinical experiments and zebrafish studies [16]. Moreover, these exposures did not induce any sign of toxicity in the embryos while resulting in quantifiable fluorescence levels in the organism.

The spatiotemporal imaging after one hour of immersion followed by dechorionation of the zebrafish embryos showed that most of the fluorescent compounds were associated with the chorion or accumulated in the perivitelline space. However, this observation does not reflect a real uptake of the compounds by the developing embryo, possibly resulting in false-negative results when toxicity or teratogenicity testing is performed. Only in the case of the most lipophilic compound (Cy3A, Log D: 1.73) could a fast intrabody accumulation be observed, implying that the compound readily passed the vitelline membrane and penetrated the embryonic cells.

The images further show that, especially when compared to the fluorescence observed after IY microinjections, most of the less lipophilic compounds were not taken up well by the embryos, even after longer exposure periods (up to $72 \mathrm{~h}$ ). Our results therefore are in line with a previous study that concluded that lipophilic dyes are better absorbed from the aqueous environment than less lipophilic ones during zebrafish embryo development [10].

Of interest, immediately after the IY microinjection a flow of some fluorescent dyes could be observed in the direction of the embryonic cells. This process is probably driven by the dynamic ooplasmic streaming that takes place in the early embryonic development [17]. However, a major part of the dyes stayed localized to the microinjection site that was redistributed over the non-yolk body parts during the following hours. The relative distribution (RD) data that compare the amount of fluorescence in the non-yolk parts with the total fluorescence present over a period of $72 \mathrm{~h}$ post-injection, indicate that about $20-30 \%$ of compound entered the rest of the embryonal body. This outcome therefore reveals that molecules are released from the dense amphiphilic environment of the yolk independent of the lipophilicity, although only slowly. The data are somewhat in contrast to the results obtained in our previous study, where a lack of intrabody distribution was 
observed in most cases after injecting the fluorescent compounds into the yolk of $3 \mathrm{dpf}$ eleuthero-embryos $[15,16]$. So, although a side-by-side comparison is lacking, it appears that administering compounds by microinjection at $0 \mathrm{dpf}$ or $3 \mathrm{dpf}$ disperses the compounds differently into the rest of the body. Clearly, more work is needed to better understand the mechanisms underlying this phenomenon. We anticipate particularly that the fast consumption of the yolk during the first days with a dynamic supply of protein, lipids, and micronutrients to the rest of the body [15], can substantially influence the biodistribution of compounds after microinjection.

Although the amount of compound microinjected in the yolk redistributed only partially, the Relative Exposure $\left(\mathrm{RE}_{10 / 2 / 72 \mathrm{~h}}\right)$ data clearly prove that drug exposures in the whole body (WB), Rest-of-Body (RoB) and yolk, were substantially higher after microinjection than after the immersion procedure for most compounds. Significantly, the outcome was different for the most lipophilic compound CY3A that reached a RE-RoB value of 1.99 after $72 \mathrm{~h}$, showing that the non-yolk body tissues and organs were more exposed to the compound after immersion than after intrayolk microinjection.

Non-compartmental pharmacokinetic analysis was also performed, followed by a QSPkR assessment, revealing that $R E_{\mathrm{WB} 10 / 2 / 72 \mathrm{~h}}$ and the $R E_{\mathrm{RoB} 10 / 2 / 72 \mathrm{~h}}$ were consistently estimated by the lipophilicity level of the compounds, whereas the estimation of $\mathrm{RE}_{\text {Yolk10/2/72h }}$ required additional molecular descriptors, i.e., TPSA, MR and the interaction of MR with the lipophilicity level of the compounds. Compound lipophilicity has been previously identified as the most critical physicochemical property for the absorption of molecules in zebrafish $[16,18,19]$ as also found in mammals [20]. Moreover, the identification of TPSA, MR, Log D as properties that explain the relative amount of compound in the zebrafish yolk can be related to the lipidic character and the rheological properties of the yolk. TPSA and MR are molecular descriptors containing information about the compounds' polarizability, thus accurately taking into account electronic parameters that have an effect on chemical-biological interactions [19-26]. This understanding aligns with the biochemical composition of the yolk, which is different from RoB [15].

\section{Materials and Methods}

\subsection{Zebrafish}

Adult wildtype (AB) zebrafish (Danio rerio) were maintained at $28.5^{\circ} \mathrm{C}$ on a $14 / 10 \mathrm{~h}$ light/dark cycle according to standard zebrafish aquaculture conditions [24]. The fish were fed with a commercial fish diet twice a day and live food (Artemia salina) once a day. The embryos were collected from natural spawning and raised in Danieau's solution $0.3 \times\left(17 \mathrm{mM} \mathrm{NaCl}, 0.2 \mathrm{mM} \mathrm{KCl}, 0.18 \mathrm{mM} \mathrm{Ca}\left(\mathrm{NO}_{3}\right)_{2}, 0.12 \mathrm{mM} \mathrm{MgSO}_{4}\right.$ and $1.5 \mathrm{mM}$ HEPES buffer $\mathrm{pH}$ 7.1-7.3) [25].

\subsection{Fluorescent Compounds}

Fluorescent compounds were acquired from Lumiprobe (Hannover, Germany): alkyne cyanine-based dyes: S-CY3A (CAS No. A13B0), S-CY5.5A (CAS No. A73B0), S-CY5A (CAS No. A33B0) CY3A (CAS No. A10B0), and alkyne xanthene-based dyes: FAMA (CAS No. A41B0), TAMRA (CAS No. A71B0) and R6GA (CAS No. A52B0). These compounds represent a wide range of $\log \mathrm{D}$ values that were determined experimentally before [16], as listed in Table 4 . This table also includes molecular descriptors of the compounds as calculated by the SwissADME platform [26]. All compounds were dissolved in DMSO $(99.9 \%)$ and frozen as $10 \mathrm{mM}$ stock solutions at $-20^{\circ} \mathrm{C}$. 
Table 4. Molecular descriptors of the fluorescent compounds and the experimentally determined Log D values.

\begin{tabular}{|c|c|c|c|c|c|c|c|}
\hline $\begin{array}{c}\text { Compound } \\
\text { NO. }\end{array}$ & $\begin{array}{c}\text { S-Cyanine } 3 \\
\text { (S-CY3A) } \\
1\end{array}$ & $\begin{array}{c}\text { S-Cyanine } 5.5 \\
\text { (S-CY5.5A) } \\
2\end{array}$ & $\begin{array}{c}\text { S-Cyanine 5A } \\
\text { (S-CY5A) } \\
3\end{array}$ & $\begin{array}{c}\text { Fam A, 5-Isomer } \\
\text { (FAMA) } \\
4\end{array}$ & $\begin{array}{c}\text { Tamra A 5-Isomer } \\
\text { (TAMRA) } \\
5\end{array}$ & $\begin{array}{c}\text { Rhodamine 6g } \\
\text { 6-Isomer (R6GA) } \\
6\end{array}$ & $\begin{array}{c}\text { Cyanine } 3 \\
\text { (CY3A) } \\
7\end{array}$ \\
\hline $\begin{array}{c}\mathrm{MW} \\
\mathrm{g} / \mathrm{mol}\end{array}$ & 691.9 & 1054.36 & 547.79 & 413.38 & 467.52 & 462.6 & 530.14 \\
\hline Rotor & 13 & 18 & 11 & 3 & 6 & 7 & 10 \\
\hline HBA & 7 & 13 & 1 & 6 & 4 & 2 & 1 \\
\hline HBD & 1 & 1 & 0 & 3 & 1 & 1 & 1 \\
\hline MR & 180.42 & 241.21 & 185.18 & 109.52 & 135.27 & 144.59 & 169.95 \\
\hline TPSA $\AA^{2}$ & 152.68 & 256.18 & 23.32 & 105.09 & 88.62 & 38.33 & 35.35 \\
\hline $\log D$ & -1.96 & -1.68 & -0.72 & -0.14 & 0.46 & 1.07 & 1.73 \\
\hline
\end{tabular}

\subsection{Fluorescent Compound Treatments}

Immersion treatment: zebrafish embryos at the two-cell stage were immersed in Danieau's medium containing the compound. A compound concentration of $10 \mu \mathrm{M}$ and DMSO concentration of $0.1 \%(\mathrm{v} / \mathrm{v})$ in a volume of $5 \mathrm{~mL}$ was used per well (6-well-plates). At $72 \mathrm{~h}$, the zebrafish medium containing the compound was removed, and the animals rinsed $3 \times$ with Danieau's medium. Next, the embryos were kept for another $48 \mathrm{~h}$ in Danieau's medium supplemented with DMSO $(0.1 \%, \mathrm{v} / \mathrm{v})$ in the absence of compound. In the case of control experiments, the embryos were exposed to Danieau's medium supplemented with DMSO $(0.1 \%, \mathrm{v} / \mathrm{v})$.

Intrayolk microinjection (IY): zebrafish embryos at the two-cell stage were positioned in a Petri dish at room temperature. IY microinjection was performed according to Rosen (2009) [12], using glass needles fitted to a micromanipulator (MM-33) connected to a gas pressure microinjector (Eppendorf Femtojet AG, Hamburg, Germany). The glass capillaries (W/FIL 1.0MM 4 in TW 100F-4) were pulled (Sutter Instrument CO. Model P-87 Cat N B100-58-15 Filament: FB330B-B320B, Novato, CA, USA) using program 5 (Heat 829, Pull 158, Vel 100, Time 150). Needles were filled with compounds dissolved in the vehicle (DMSO/saline (1:1)), placed under the microscope, and using forceps, the tip was cut off in a manner to allow for a consistent volume to be injected. Afterwards, the embryos were transferred to 6-well plates. Control embryos were exposed to vehicle only.

\subsection{Spatiotemporal Fluorescence Imaging}

Animals were kept in dark conditions in an incubator at $28.5{ }^{\circ} \mathrm{C}$ for defined timeperiods $(0.25,1,3,6,24,48,72,96$, and $120 \mathrm{~h})$ before image analysis in a dark room. To acquire images, a Leica MZ10F fluorescent stereomicroscope (Leica Microsystems Inc., Buffalo Grove, IL, USA) with a $4.0 \times$ planapochromatic objective (10447243) was used, equipped with a Digital Color Camera Leica DFC310 FX (Software LAS 4.13, Leica Microsystems Inc., Buffalo Grove, IL, USA). Filter sets were GFP 10446222 in the case of compound FAMA, dSRED 10447079 in the cases of S-CY3A, CY3A and TAMRA, and CY5 10446366 in the cases of S-CY5.5A and S-CY5A. Previous to fluorescence imaging, the embryos were dechorionated (up to $72 \mathrm{hpf}$ ) and immobilized by hypothermia, rinsed three times with Danieau's medium, and positioned latero-lateral (right lateral recumbency) on a single cavity glass slide and covered by a drop of agarose $(0.1 \%)$. Then, using MetaMorph (Microscopy Automation and Image Analysis Software V.7.8.00, Molecular Devices, LLC., San Jose, CA, USA) and by manual delineation of the whole-body (WB) (up to $120 \mathrm{~h}$ ) and yolk sac contours (up to $72 \mathrm{~h}$ ) of the zebrafish embryos the fluorescence in the selected area was quantified as integrated fluorescence intensity (RFU) [16]. The RFU values of the non-yolk compartment (i.e., RoB: rest of body) was assessed by subtracting the yolk results from the corresponding integrated fluorescence intensities found in the WB. 


\subsection{Non-Compartmental Pharmacokinetic Analysis}

In order to determine the degree of tissue exposure following administration of the fluorescent compounds, we calculated the AUC as the pharmacokinetic parameter of the non-compartmental analysis. The area under a plot of the fluorescence intensity $\left(R F U_{T}\right)$ versus time is referred to as the area under the (zero moment) curve $A U C . R F U_{T}$ represents the relative amount of compound present in the ZFE by measuring the integrated fluorescence intensity of delineated contours in fluorescence images (Figure 2). The AUC was then determined by summing the incremental area of subsequent trapezoids [27]. Therefore, the linear trapezoidal method for calculation of the AUC is (Equation (1)):

$$
A U C_{0.25}^{72 h}=\sum_{i=0.25 \mathrm{~h}}^{n} \frac{R F U_{T i}+R F U_{T i+1}}{2} . \Delta t,
$$

where RoB $t=t_{0.25^{\prime}+1}-t_{0.25}$ and $t_{72 \mathrm{~h}}$ denotes the time of the last measured $R F U_{T}$. In some cases, observations were censored and excluded from the analysis, due to possible fluorescence quenching effect of compounds that remains at the site of the intrayolk microinjection.

We further calculated the Relative Exposure $\left(R E_{10 / 2}\right)$ by comparing the $A U C_{0-120 \mathrm{~h}}$ after immersion $(10 \mu \mathrm{M})$ with the $A U C_{0-120 \mathrm{~h}}$ after IY microinjection $(2 \mathrm{mg} / \mathrm{kg})$, as defined in Equation (2) [16]:

$$
R E_{10 / 2 / \mathrm{h}}=\frac{A U C_{I m m}}{A U C_{I n j}} .
$$

\subsection{Statistical Analysis}

The non-compartmental and the QSPkR analyses were performed using JMP (Version 15.1. SAS Institute Inc., Cary, NC, USA). We ran the QSPkR analysis identifying the association among $R E$, with the experimental $\log \mathrm{D}$ value and some molecular descriptors applying a multiple linear regression analysis.

\section{Conclusions}

In conclusion, by using a fluorescence-based approach in this study, we show that a $72 \mathrm{~h}$-long immersion of embryos starting at a two-cell stage results in an intrabody exposure which is similar or higher than that seen after a $2 \mathrm{mg} / \mathrm{kg}$ intrayolk microinjection, at least in the case of a lipophilic compound (log D: 1.73). In contrast, zero to low intrabody exposure was reached after immersion of the embryos with less lipophilic compounds, possibly resulting in a false-negative outcome in screening programs. In the latter case IY microinjection, a technical procedure that can be easily automated, is highly recommended $[12,22,23]$. Alternatively, higher immersion concentrations than the one used in this study could possibly be deployed in order to increase intrabody exposure to compounds. Future studies should consider examining the relationship between immersion concentrations and the relative uptake in ZFE, essential information that is presently missing in literature.

Supplementary Materials: The following are available online at https:/ /www.mdpi.com/article/10.3 390/ph14060576/s1, Figure S1: Representative images of embryos showing the flow of some fluorescent dyes from the yolk (bottom) to the proliferating embryonic cells (top) after intrayolk microinjections (2 $\mathrm{mg} / \mathrm{kg}$ ) in compounds S-CY3A (1 h), FAMA $(0.25 \mathrm{~h})$, TAMRA $(1 \mathrm{~h})$, and CY3A $(1 \mathrm{~h})$.

Author Contributions: Conceptualization, M.G., A.N., N.D.C., M.L., P.A. and P.A.M.d.W.; methodology, M.G., P.A. and P.A.M.d.W.; software, M.G.; validation, M.G., P.A. and P.A.M.d.W.; formal analysis, M.G.; investigation, M.G. and J.M.; resources, A.N., N.D.C., M.L. and P.A.M.d.W.; data curation, M.G.; writing—original draft preparation, M.G.; writing—review and editing, A.N., P.A. and P.A.M.d.W.; visualization, M.G.; supervision, P.A.M.d.W.; project administration, A.N.; funding acquisition, M.G., A.N. and P.A.M.d.W. All authors have read and agreed to the published version of the manuscript. 
Funding: This research was funded by Fundación CeiBA, Colombia “Rodolfo Llinás para la promoción de la formación avanzada y el espíritu científico en Bogotá" and by L'Oréal (contract C150353).

Institutional Review Board Statement: All procedures were carried out according to the Declaration of Helsinki and the guidelines of the European Community Council Directive 2010/63/EU, implemented in 2020 by the Commission Implementing Decision (EU) 2020/569 and all the relevant ethical regulations from the Ethics Committee of the University of Leuven (Ethische Commissie van de KU Leuven, approval number ECD 027/2019) and from the Belgian Federal Department of Public Health, Food Safety and Environment (Federale Overheidsdienst Volksgezondheid, Veiligheid van de Voedselketen en Leefmilieu, approval number LA1210261).

Informed Consent Statement: Not applicable.

Data Availability Statement: The data supporting reported results are digitally archived and can be obtained from the corresponding author upon reasonable request.

Conflicts of Interest: The authors declare no conflict of interest.

\section{References}

1. Sewell, F.; Edwards, J.; Prior, H.; Robinson, S. Opportunities to Apply the 3Rs in Safety Assessment Programs. ILAR J. 2016, 57, 234-245. [CrossRef]

2. Cox, A.G.; Goessling, W. The lure of zebrafish in liver research: Regulation of hepatic growth in development and regeneration. Curr. Opin. Genet. Dev. 2015, 32, 153-161. [CrossRef]

3. OECD. Test No. 236: Fish Embryo Acute Toxicity (FET) Test; OECD: Paris, France, 2013.

4. Busquet, F.; Strecker, R.; Rawlings, J.M.; Belanger, S.E.; Braunbeck, T.; Carr, G.J.; Cenijn, P.; Fochtman, P.; Gourmelon, A.; Hübler, N.; et al. OECD validation study to assess intra- and inter-laboratory reproducibility of the zebrafish embryo toxicity test for acute aquatic toxicity testing. Regul. Toxicol. Pharmacol. 2014, 69, 496-511. [CrossRef]

5. Rihel, J.; Ghosh, M. Zebrafish. In Drug Discovery and Evaluation: Pharmacological Assays; Hock, F., Ed.; Springer International Publishing: Cham, Switzerland, 2016; pp. 4071-4155.

6. Hisaoka, K.K. Microscopic Studies of the Teleost Chorion. Microsc. Soc. 1958, 77, 240-243. [CrossRef]

7. Brox, S.; Ritter, A.P.; Küster, E.; Reemtsma, T. Influence of the perivitelline space on the quantification of internal concentrations of chemicals in eggs of zebrafish embryos (Danio rerio). Aquat. Toxicol. 2014, 157, 134-140. [CrossRef]

8. Hamm, J.T.; Ceger, P.; Allen, D.; Stout, M.; Maull, E.A.; Baker, G.; Zmarowski, A.; Padilla, S.; Perkins, E.; Planchart, A.; et al. Characterizing sources of variability in zebrafish embryo screening protocols. ALTEX 2019, 36, 103-120. [CrossRef]

9. Henn, K.; Braunbeck, T. Dechorionation as a tool to improve the fish embryo toxicity test (FET) with the zebrafish (Danio rerio). Comp. Biochem. Physiol. C Toxicol. Pharmacol. 2011, 153, 91-98. [CrossRef]

10. De Koning, C.; Beekhuijzen, M.; Tobor-Kapłon, M.; de Vries-Buitenweg, S.; Schoutsen, D.; Leeijen, N.; van de Waart, B.; Emmen, H. Visualizing Compound Distribution during Zebrafish Embryo Development: The Effects of Lipophilicity and DMSO. Birth Defects Res. B Dev. Reprod. Toxicol. 2015, 104, 253-272. [CrossRef] [PubMed]

11. Rawson, D.M.; Zhang, T.; Kalicharan, D.; Jongebloed, W.L. Field emission scanning electron microscopy and transmission electron microscopy studies of the chorion, plasma membrane and syncytial layers of the gastrula-stage embryo of the zebrafish Brachydanio rerio: A consideration of the structural and functional relationships with respect to cryoprotectant penetration. Aquac. Res. 2000, 31, 325-336. [CrossRef]

12. Spaink, H.P.; Cui, C.; Wiweger, M.I.; Jansen, H.J.; Veneman, W.J.; Marín-Juez, R.; de Sonneville, J.; Ordas, A.; Torraca, V.; van der Ent, W.; et al. Robotic injection of zebrafish embryos for high-throughput screening in disease models. Methods 2013, 62, 246-254. [CrossRef]

13. Ng, A.N.; de Jong-Curtain, T.A.; Mawdsley, D.J.; White, S.J.; Shin, J.; Appel, B.; Dong, P.D.; Stainier, D.Y.; Heath, J.K. Formation of the digestive system in zebrafish: III. Intestinal epithelium morphogenesis. Dev. Biol. 2005, 286, 114-135. [CrossRef]

14. Silfvast, W.T. Laser Fundamentals, 2nd ed.; Cambridge University Press: Cambridge, UK, 2004.

15. Fraher, D.; Sanigorski, A.; Mellett, N.A.; Meikle, P.J.; Sinclair, A.J.; Gibert, Y. Zebrafish Embryonic Lipidomic Analysis Reveals that the Yolk Cell Is Metabolically Active in Processing Lipid. Cell Rep. 2016, 14, 1317-1329. [CrossRef] [PubMed]

16. Guarin, M.; Faelens, R.; Giusti, A.; De Croze, N.; Léonard, M.; Cabooter, D.; Annaert, P.; de Witte, P.; Ny, A. Spatiotemporal imaging and pharmacokinetics of fluorescent compounds in zebrafish eleuthero-embryos after different routes of administration. Sci. Rep. 2021, 11, 12229. [CrossRef] [PubMed]

17. Shamipour, S.; Kardos, R.; Xue, S.L.; Hof, B.; Hannezo, E.; Heisenberg, C.P. Bulk Actin Dynamics Drive Phase Segregation in Zebrafish Oocytes. Cell 2019, 177, 1463-1479. [CrossRef] [PubMed]

18. Padilla, S.; Corum, D.; Padnos, B.; Hunter, D.L.; Beam, A.; Houck, K.A.; Sipes, N.; Kleinstreuer, N.; Knudsen, T.; Dix, D.J.; et al. Zebrafish developmental screening of the ToxCast ${ }^{\mathrm{TM}}$ Phase I chemical library. Reprod. Toxicol. 2012, 33, 174-187. [CrossRef]

19. Long, K.; Kostman, S.J.; Fernandez, C.; Burnett, J.C.; Huryn, D.M. Do Zebrafish Obey Lipinski Rules? ACS Med. Chem. Lett. 2019, 10, 1002-1006. [CrossRef] 
20. Winiwarter, S.; Ridderström, M.; Ungell, A.L.; Andersson, T.B.; Zamora, I. Use of Molecular Descriptors for Absorption, Distribution, Metabolism, and Excretion Predictions. In Comprehensive Medicinal Chemistry II.; Triggle, D.J., Taylor, J.B., Eds.; Elsevier Ltd.: Amsterdam, The Netherlands, 2007; Volume 5, pp. 531-554.

21. Tandon, H.; Ranjan, P.; Chakraborty, T.; Suhag, V. Polarizability: A promising descriptor to study chemical-biological interactions. Mol. Divers. 2021, 25, 249-262. [CrossRef]

22. Wang, W.; Liu, X.; Gelinas, D.; Ciruna, B.; Sun, Y. A fully automated robotic system for microinjection of zebrafish embryos. PLoS ONE 2007, 2, e862. [CrossRef]

23. Cordero-Maldonado, M.L.; Perathoner, S.; van der Kolk, K.J.; Boland, R.; Heins-Marroquin, U.; Spaink, H.P.; Meijer, A.H.; Crawford, A.D.; de Sonneville, J. Deep learning image recognition enables efficient genome editing in zebrafish by automated injections. PLoS ONE 2019, 14, e0202377. [CrossRef]

24. Avdesh, A.; Chen, M.; Martin-Iverson, M.T.; Mondal, A.; Ong, D.; Rainey-Smith, S.; Taddei, K.; Lardelli, M.; Groth, D.M.; Verdile, G.; et al. Regular care and maintenance of a zebrafish (Danio rerio) laboratory: An introduction. J. Vis. Exp. 2012 , e4196. [CrossRef]

25. Danieau's Solution $(30 \times)$. Cold Spring Harbor Protocols. 2011. Available online: http://cshprotocols.cshlp.org/content/2011/7 /pdb.rec12467.full (accessed on 13 June 2021).

26. Daina, A.; Michielin, O.; Zoete, V. SwissADME: A free web tool to evaluate pharmacokinetics, drug-likeness and medicinal chemistry friendliness of small molecules. Sci. Rep. 2017, 7, 42717. [CrossRef] [PubMed]

27. Gabrielsson, J.; Weiner, D. Non-compartmental analysis. Methods Mol. Biol. 2012, 929, 377-389. [CrossRef] 